\title{
Working-memory prefrontal model for cognitive flexibility in task-switching and selection
}

\author{
Julien Abrossimoff, Alexandre Pitti, Philippe Gaussier \\ ETIS UMR 8051 CY University, ENSEA, CNRS F-95000 Cergy, France. \\ Email: firstname.lastname@cyu.fr
}

\begin{abstract}
We propose a working-memory model inspired by the prefrontal cortex for flexible cognitive tasks. Our neural model categorizes contexts depending on the task features and selects the most appropriate one relative to inputs. Output errors led to a reevaluation of the context confidence level and a shift to another or the creation of a new one. Tests on the Wisconsin card task show the relevance of our model. Impairments of brain parts that regulate the neuromodulators for context evaluation or output selection produces similar prefrontal disorder and degeneration found in the brain literature.
\end{abstract}

Index Terms - top-down learning, bottom-up learning, rules inference, task-set shifting, working memory

\section{INTRODUCTION}

The ability to maintain a behaviour during a period of time or to switch between multiple concepts is very important for achieving complex tasks that require to plan and compose with multiple sensory information. In this paper we will present a neural architecture for learning and switching contextual rules in the Wisconsin card sorting test (WCST).

\section{A. Neurosciences studies}

In neuropsychology, there are various experiences to test the cognitive ability to maintain a behaviour of a patient. They are designed to show the cognitive effects of neurological disorders (memory impairments, executive functions, ...). In case of task switching, we can cite the Wisconsin card sorting test, which permits to measure the ability of a subject to create a contextual rule and to maintain it : if we present different cards with different shapes, colors or values, we need to be able to select one of these characteristics (a rule associated to a context) to select one card [17].

Neurosciences explain how effects found by neuropsychologists in task switching depends on the exchanges between BG (basal ganglia) and PFC (prefrontal cortex). They are necessary for executive functions such as task shifting. For instance, studies have shown that people with Parkinson or Schizophrenia diseases or addictions have issues in dopaminergic and serotonic pathways [2]. These deregulations in the dopaminergic system imply a sensitivity to noise in rewardrelated cognition, which has for effect a deficit in set shifting tasks, and more generally in cognitive processes [10]. In frontal cortex, studies show the anterior cingulate cortex (ACC) is responsible for high cognition processing in its dorsal part [7], [8], a lesion in ACC will result in a deficit in error detection processes [3]. Futhermore, studies also present that people with Tourette syndrome, schizophrenia, which both imply an inhibition of social contexts, have different level of cholinergic and serotonin neuromodulators activities compared to healthy subjects [21]. These neuromodulators have shown their implication in the inhibition and the uncertainty in context selection and associated rules [14], [16], [18], [28].

The study of these different diseases or impairments suggests that the presence of an inhibition mechanism is vital in the decision making process and in action selection, it also demonstrates the importance of the basal ganglia - frontal cortex loop for executive functions and particularly for the creation and the maintainance of semantic/contextual rules [24], which permits to achieve complex tasks such as Hanoi towers or Wisconsin card sorting. These rules are dynamic, they are created along a learning process, by accumulating experience in different contexts. They are also plastic : the association can change through time like the card association in the Wisconsin test. If we select a rule in excitatory selection, it is a situation that has no impact for the plausibility of the rule for the current context because the previous associated rule is not inhibited. Conversely, in a case of rule inhibition, we are sure of its non-validity for this context. There is some kind of asymmetry between the two mechanisms. The inhibition mechanism, in a case of random exploration is better in terms of computational cost.

Similarly, in order to plan a complex task in our environment, we need to order / prioritize our sensorimotor level or maintain purpose with a higher level of abstraction.

Prefrontal neurons have shown their abilities to maintain information for many seconds or until the task is succeeded in the working-memory [4]. These specificities of the working memory permit us to maintain information of different granularity (visual goal, error during a task ...) for a long period of time [15] and permits to maintain a visual goal even if the visual cue is no longer present [26]. The update in the working memory is based on a input gating control that permits to open the gate when a useful information is available and then reinforce the associated activity or to close it when an irrelevant information is presented [11]. Based on FMRi studies, basal ganglia appears, with dopaminergic projections, to be the main mediator of this input gating system [5], [13]. According to the top-down biased competition model [6], [31], the evidence of an output gating control is enlighted, this mechanism permits to activate specific neurons in the dorsal pre-premotor cortex [9]. 


\section{B. State of the art studies on prefrontal models}

In state of art, gated networks are present on various domains : in image processing [22], in robotics for learning to write numbers [12] or to learn multimodal representations [1] and even for the learning of higher functions like affordances [23]. Hasselmo proposed a model of gating associated learning for the learning of rules which are composed of multiple combinations of input patterns, and shows the importance of gating in learning processes compared to classical hebbian learning association [19]. Tani shows the principles of operation of information processing at a nodal point in the PFC, or through a cascade of nodes that processes information successively to achieve a behavioral goal [29]. In state of art, the action is often not included in models, the resolution of problems like Wisconsin card sorting test, Stroop test or the learning of physical rules [30] are realised on a strictly visual point of view and with discrete time. In the learning of rules, we can cite the paper of Rougier [27], which presents an architecture capable of learning rules that depend on various visual cues (shape, color, position, size), or the paper of Pitti et al. [25] for the learning of tasks in motor control situation.

\section{A bio-inspired model}

After we describe our neural network that is inspired on the characteristics of the BG-PFC loop and working memory effects. This approach has multiple advantages :

- To predict a rule in an instantaneous way : even if the prediction is wrong, it will be refine during the experiment.

- An architecture of working memory that maintain information (rule,context,...) during a long period of time.

We will present the results for dynamical context/rule switching and the push up of an inferred rule. We show that few contexts are required to solve the task and then permitted a fast switching when the association rule changes. We will also show the impact of impairments on the different simulated part of the BG-PFC loop (ACC and BG). We will then discuss the relevance of our model in real robotic tasks and its utility for more executive function tasks such as ( planning, problem solving, ...)

\section{ARChitecture AND NEURAL MECHANISMS}

\section{A. Visual and integrative modules}

For visual and the multimodal modules resp. represented with red and green dashed blocks in Fig. 1 and corresponding to the visual and parietal systems. We assume that our system has access to an analyzed image by the visual cortex and has then three different channels for the three modalities (value, shape, color of the card). Each of these channels are categorized separately to reduce the input dimension with factored functions or basis functions, $f_{S}, f_{C}$ and $f_{V}$ where $S, C$ and $V$ are the inputs resp. for shape, color and value following the Eq.(1) and $W$ their associated weights.

$$
\begin{aligned}
& f_{S}=\sum W_{S} * S \\
& f_{C}=\sum W_{C} * C \\
& f_{V}=\sum W_{V} * V
\end{aligned}
$$

They are modeled with feed-forward neural networks and they are then merged into a multimodal node to represent a conjunction of the three modalities [1].

\section{B. Frontal system compound model}

In this section we detail the different components of our model of frontal cortex, purple dashed block in Fig. 1

1) Context recruitment: The context recruitment module is based on a variation of K-mean algorithm, it recruits new neurons based on an adaptive vigilance parameter. The output of context module is given by Eq.(2) where $Y$ represents all recruited context for a particular task and $E_{i}$ represents a particular multimodal node. A new node is recruited if vigilance parameter is greater than the maximum value $Y_{\max }$ of $Y$ Eq.(3)

$$
\begin{gathered}
Y=\sum W_{Y} * E_{i} \\
\Delta W_{i j}= \begin{cases}E_{i} & \text { if } Y_{\text {jmax }}<\text { vigilance } \\
0 & \text { otherwise }\end{cases}
\end{gathered}
$$

2) ACC: ACC is the mediator of the context recruitment and context competition. It monitors the error detection and conflictual situations. ACC works like a control gate : if it has no activity the context remains the same, otherwise if ACC has activity the system need to initiate a new competition or to recruit a new context. To know if the system needs a new competition or to recruit a new context, ACC looks what is available in the short-term memory(STM). If the STM is empty (i.e there is no context recruited for the task or all already recruited contexts are not satisfying the task), the ACC sends a signal (vigilance) to the context recruitment module to recruit a new context.

Otherwise, a competition is initiated, this process is based on a winner takes all mechanism, from all available contexts in the STM. During competition if ACC detects error it can inhibit the selected context in the STM to avoid perseverance in context selection if error is detected following Eq.(4).

$$
Y_{j}= \begin{cases}Y_{j}-1 & \text { if error }>0 \\ Y_{j} & \text { otherwise }\end{cases}
$$

3) Working memory module: Hebb's rule describes with a differential equation how we can represent synaptic plasticity and the adaptation of neurons during learning process. This result that the weight between two neurons increases if the two neurons activate simultaneously and decrease otherwise [20]. In our case it will serve to implement a dynamical and plastic working memory. The input information of this working memory corresponds to the winner context. The system will associate this context with working memory's activities $S_{j}$. This bounding between WM activities and contexts form contextual rules.

The basal ganglia contributes to working memory input gating : a good selection of working memory output will reinforce the input noise which result an increase of the synaptic weights between the selected context and the winner neuron of the working memory. An error of prediction will 


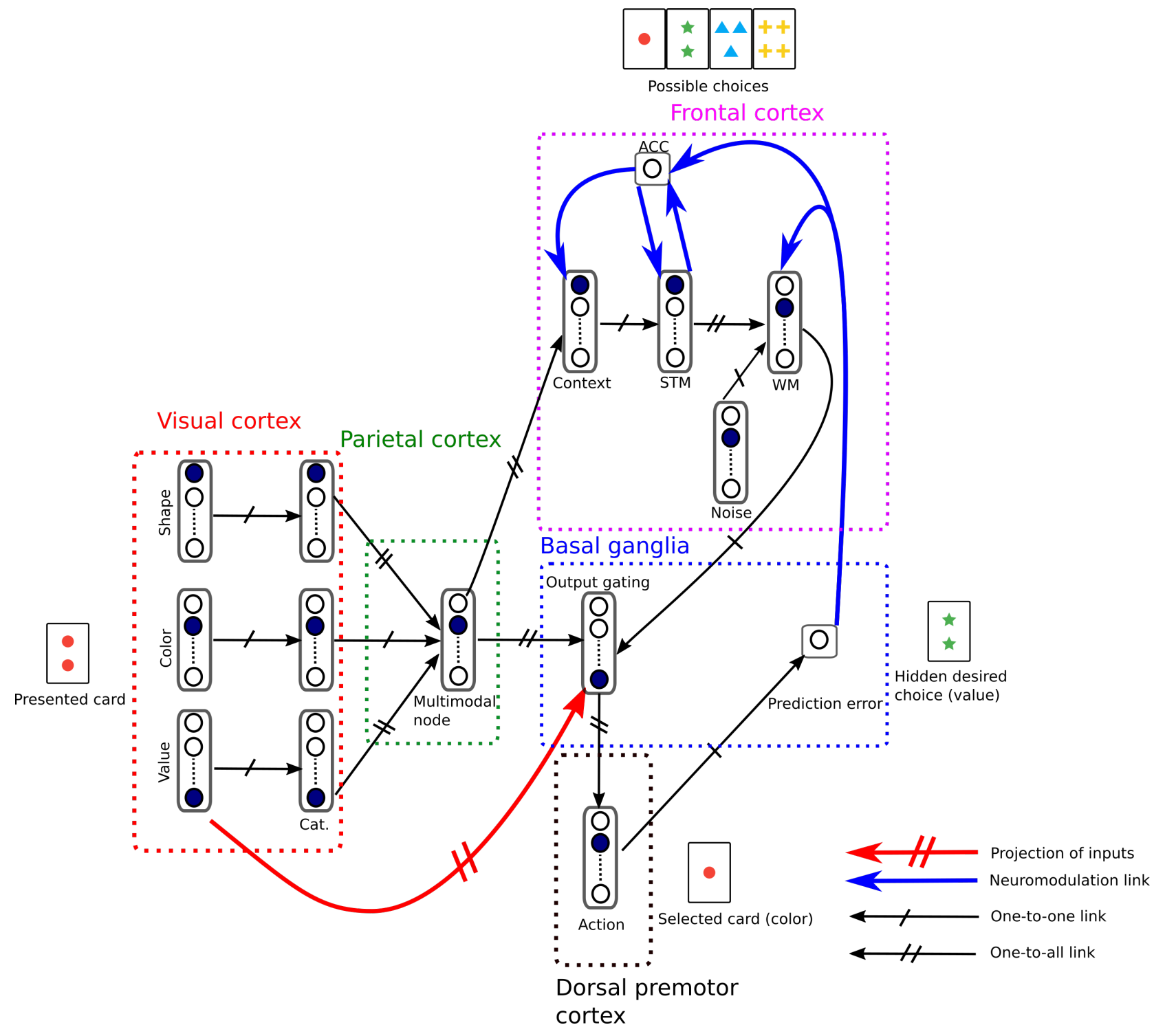

Fig. 1. Neural network for learning contextual rules. The network learns from the conjunction of the different characteristics of a game card (color, value, shape) to infer a contextual sorting rule (by color, by shape or by value). For that it needs to recruit different contexts if necessary or to compete between already recruited contexts, this part is modulate by the ACC, that play a mediator role. The working memory corresponds to which rule is maintained or not, it's input flow (i.e inhibition or reinforcement of the current selected neuron) is handled by the basal ganglia part depends on prediction error. The output gating of the WM corresponds litterally to gates that are activated or not by the WM to let through one or multiple sensory signal(s).

decrease the synaptic weights for inhibiting these output of the working memory for the current context, see Eq.(6). This mechanism, after exploration, stabilizes a random neuron and specialize it for particular rule. The output of the working memory $Y$ is the product between input variables $E_{i}$ and the weights $W_{i j}$ Eq.(5).

The different parameters for the input gating of the working memory are $\epsilon, \alpha$ and $\beta$, they are local neuromodulations, they represent resp. the reinforcement signal, the passive decay in neuronal activity and the decay if the input is not active.

$$
Y_{j}=\sum_{i} \sum_{j} W_{i j} E_{i}
$$

TABLE I

VALUES OF THE DIFFERENT PARAMETERS OF THE WM

\begin{tabular}{|l|l|}
\hline noise rate & 0.5 \\
\hline$\epsilon$ & 0.8 \\
\hline$\beta$ & 0.4 \\
\hline$\alpha$ & 0.1 \\
\hline
\end{tabular}

$$
\Delta W_{i j}=\epsilon * E_{i} * S_{j}-W_{i j}\left(\alpha+E_{i} * \beta\right)
$$

4) Output gating: The output gating permits, in function of neurons active in the WM to select one or multiple inputs and to block other ones.

In the case of the Wisconsin card sorting, the output gating 
unit will only learn to let go one modality and block the others. Each neuron of the WM is connected to a gate, and the activity of this neuron determines if the gate is open or close. If the gate is opened the output is then a recopy of the connected modality, otherwise the gate is close and the output is zero.

\section{Dorsal premotor cortex (DPC)}

We made the assumption that the dorsal premotor cortex (black dashed block in Fig. 1) has already learned the association between a visual cue (value,shape,color) and the corresponding action (association between colors, values, ...). DPC is then able, in function of the selected rule to place correctly the card to the corresponding deck.

\section{Basal ganglia}

We model the prediction error mechanism of the basal ganglia, which is represented with blue dashed block in Figure 1. The experimenter has a desired rule (sort by color for example), the prediction error will compute the difference between the current output given by where the card is placed (i.e which association is selected) by the system and the signal given by the experimenter : true or false for the current association. Depends on activity of the group, an inhibition flow will be send to the system if an error is detected, otherwise an reinforcement flow will be send.

\section{EXPERIMENTAL SETUP}

We choose Wisconsin card sorting test to analyze the performances of our WM system for decision marking. Four cards are disposed in line, each card corresponds to different visual stimulus (number, shape, color).There are four different stimulus for each characteristics, see Table. II. Cards will in a random manner be presented to the neural network randomly. There is 64 cards in the database, which is corresponds the $4^{3}$ possibilities of values, shapes and colors. We give as inputs to the network a card of the Wisconsin card sorting test, this card is decomposed into three different channels by the visual cortex, each channel corresponds to resp. the value, the color and the shape of the card. Each of these channels are then categorize separately into feature functions $f_{V}, f_{C}$ and $f_{S}$ from (1) consist on 4 units each, factorizing the input space. The multimodal recruitment node is based on 10 units and represents the conjunction of the different inputs. The system based on ACC signal can dynamically recruit contexts, see part II.1 and part II.2. The desired rule given by "the teacher" is hidden to the system and only serves to modulate the system through positive or negative assessments. During learning stage, an iteration correspond to the presentation of one card, few steps are require to make the WM to converge on a valid rule.

TABLE II

VALUES OF THE DIFFERENT CARD PARAMETERS

\begin{tabular}{|l|l|}
\hline color & red, blue, green, yellow \\
\hline shape & circle, star, triangle, cross \\
\hline value & $1,2,3,4$ \\
\hline
\end{tabular}

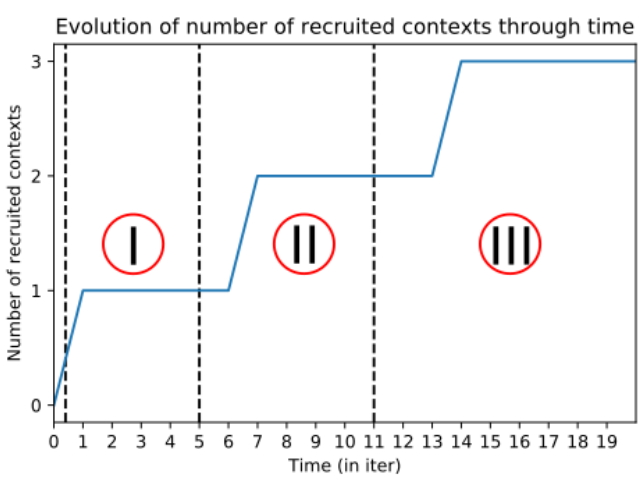

Fig. 2. Evolution through time of the number of context recruited.Each phase corresponds to the presentation of a new context to discover.

\section{RESULTS}

\section{A. In the learning of a new task}

The neural network achieves a rule inference and information maintenance through context recruitment and gating flow of information. As mentioned previously in section II.2), the ACC is responsible for context error detection and control of the competition. In our case we want to apply this mechanism during the learning of a new task, and to see how the system is able to understand that it needs the recruitment of a new context. We plot in Fig. 2, the evolution of number of contexts recruited through time for the WCST task.

As we can see at the beginning of the experiment in phase I, the system has no contexts dedicated to the WCST task, the contexts are gradually recruited through time when the ACC system has inhibated all already recruited contexts and there is still an error of prediction. Each phase corresponds to the presentation of a new context to discover. We can also see that only 3 contexts are recruited, they corresponds to the auto-organization of the system around the different sorting rules (by color, by value or by shape). The fact that the system only recruits 3 contexts for the WCST, shows us that the ACC permits to the system to be ecological in terms of context recruited for the task. In fact like ACC sends a recruitment signal for a new context only if there is still an error of prediction after the inhibition of all previously recruited contexts it avoids the recruitment of unecessary contexts. The reduced context recruited also permits to reduce the time of competition between two switching of associated rule.

In order to demonstrate the ability of the network to select a contextual rule and maintain it after the recruitment of the contexts corresponding to the WCST task, we plot in Fig. 3 the indexes of the selected rule (red dots) by the WM compared to those desired by the experimenter (blue line). A difference between the two indicates an error of prediction of the system to infer the sorting rule (i.e a bad selection of context). During these periods of error, ACC is then active and forced the system to initiate a context competition during its activation time (black signal in Fig.3). We can see that during the period 

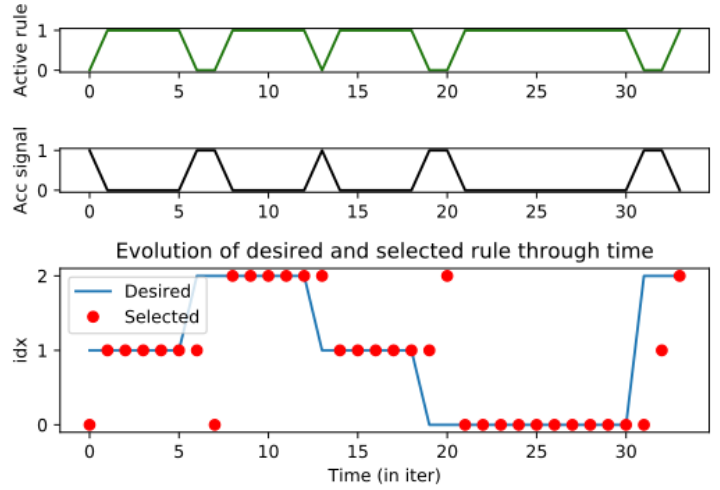

Fig. 3. Evolution of selected rules through time depending of ACC signal activation
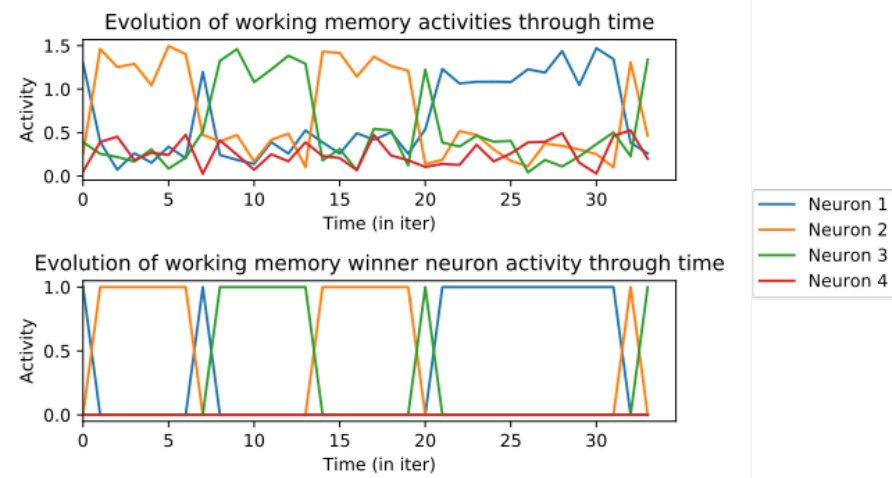

Fig. 4. Representation of activity of the working memory through time for the presentation of different rules

when ACC is not active (green line in Fig.3), the current selected rule is maintained. We can also notice that for each task switching, the system persevere only one time on its current context (the time for ACC to detect the error).

We plot in Fig. 4 the activities of the different neurons of the WM system corresponding to the different rules activated in Fig. 3. The duration of activation of the winner neurons are related to the plateau formed by indexes of selected rule (red dots) in Fig. 3. We can see that particular neurons are active for the different selected contexts, they corresponds to valid rules for a particular context. In our case only one rule is valid per context.

TABLE III

CORRESPONDENCE WM ACTIVATION/SELECTED RULE

\begin{tabular}{|l|l|}
\hline Neuron 1 & by color \\
\hline Neuron 2 & by shape \\
\hline Neuron 3 & by value \\
\hline Neuron 4 & not recruited for WCST \\
\hline
\end{tabular}

\section{B. ACC impairments}

In the previous section, we described a model for rule inference and information maintenance with a functionally

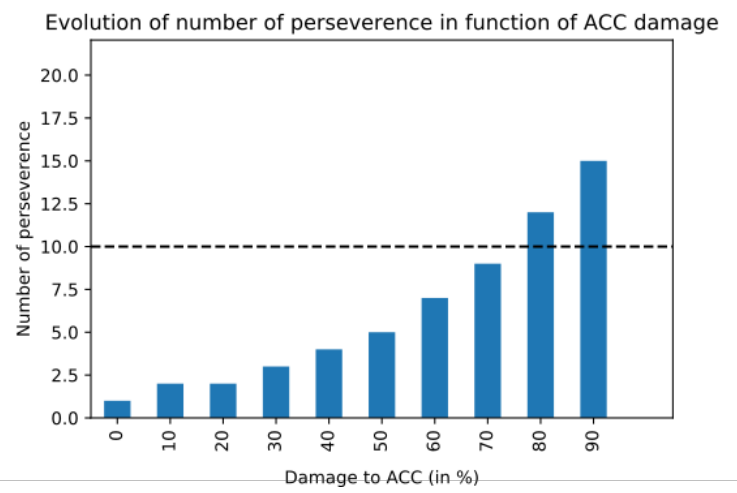

Fig. 5. Number of perseverance in a particular context depending on various damage degrees on ACC part.

ACC that permits a quick error detection and then initiate a new context competition. As we see previously, with no impairments the integration of prediction error is instantaneously and a new competition is then started. But in case of ACC impairments or schizophrenia disease, the change of context is not detected immediately, the subject has perseverance in the current task and needs some time to adapt the context. In this section, we propose to use the same architecture with the same inputs but with adding impairments of the ACC part, which will correspond to difficulties for the system to resolve contradictory rules (when the experimenter decides to change the association) and then detect a variation in contexts. The experiment aims at testing the ability of the network to infer a contextual rule on the WCST experiment with different level of ACC impairments. The result will be a delay to infer the rule compared to experiments in previous section due to perseverance in a particular context. We model the difficulty for the system to integrate the error of prediction with using a decay factor following the equation:

$$
E(t)=E(t-1) * e^{-\beta * D_{i}}
$$

where:

$$
\begin{aligned}
& \beta=\text { constant parameter }=4.5 \\
& E=\text { error of prediction } \\
& D_{i}=\text { damage level }
\end{aligned}
$$

As we can see in Fig. 5, we plot the response of the system for different damage levels from $0 \%$ (which is corresponds to healthy subject) to $90 \%$ (which is corresponds to a partial fully damage ACC) and their corresponding number of perseverance in a particular context. We retrieve the same results as section IV.A for the $0 \%$ damage (see Fig. 3), we can also notice that perseverance in specific context explode exponantially in function of ACC damage. We consider at 10 trials on the same context that the task switching is failed. The $100 \%$ damage level is not tested here, it will correspond to an uncapacity for the system to switch from a previous context. 


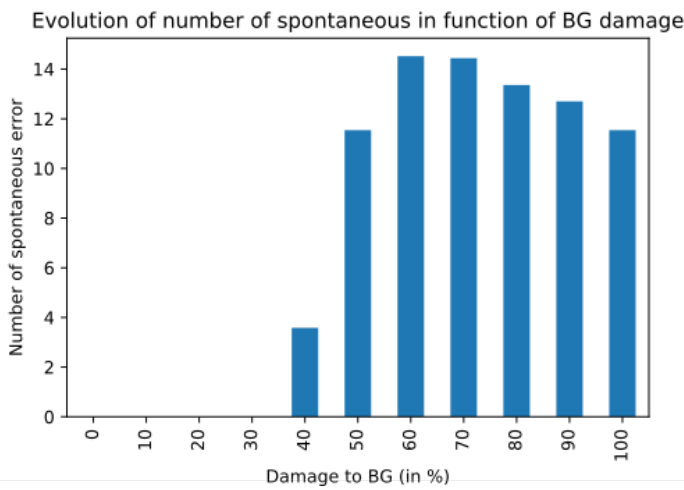

Fig. 6. Representation of the mean number of spontaneous error for 50 presentation of a rule depending on various damage degrees on BG part.

\section{BG impairments}

In this part, we use the described model that we used in section IV.A, ACC returns to fully functionnal in this section. As we see previously the BG permits the modulation of the WM system through dopamine signal, by inhibiting or reinforcing the output of the WM for a given context. In a case of healthy patient, BG stabilizes the WM for the different learned contexts, like we saw previously with Fig. 4, for the different contexts, the output of the WM is maintained. With impairments in the BG system, the selection and maintain of activities become more sensitive to noise and results to sporadic error in WM output selection even if the context selected is correct. In this section, we propose to study the effects of dopamine signal regulation, with adding impairments in the BG system. This experiment aims to show how the system behaves in situation of BG impairments. The result will show sporadic error in WM output selection. We modeling the deregulation of dopamine signal with the addition of a random white noise to the output of WM, following the equation (8)

$$
S=S+\alpha * N
$$

where:

$$
\begin{aligned}
& \alpha=\text { damage level } \\
& S=\text { output of the WM } \\
& N=\text { white noise }
\end{aligned}
$$

We simulate different range of impairments, by playing on the amplitude $\alpha$ of the noise, a null noise corresponds to healthy patient and a maximum amplitude factor corresponds to a fully damage BG system. In Fig. 6, we plot the mean number of sporadic errors in WM output selection for different level of DG impairments (from $0 \%$ for an healthy patient, to $100 \%$ for a full damage BG system) for 50 WCST samples . We retrieve results from part IV.A : the output of the WM is directly selected : the noise is not enough to alter the output of the WM. From $50 \%$ to $100 \%$ of damage rate, the noise is equal or bigger than the WM signal, the system behaves with random exploration for selecting its output.

\section{CONCLUSiON}

We proposed a neuro-inspired framework of working memory for contextual rule learning by dynamically recruit contexts and dynamically associate them to one or various working memory output(s). These kind of mechanisms are well-known in neurosciences, they rely mainly on two neuromodulators cholinergic and dopamine, which are playing roles mainly in prefrontal cortex and basal ganglia. These features are ecological for learning the contexts associated to a particular task or to compete between contexts in case of task-shifting.

In future works, we will extend our work on our complete humanoid robot arm called Tino, in order to reproduce Go/No Go task or Stroop test. We also want to investigate the mechanisms behind the learning of sequence of different rules in order to solve task such as Hanoi towers.

\section{ACKNOWLEDGMENT}

This work was partially supported by grants from the EQUIPEX-ROBOTEX (CNRS), the chaire d'excellence CNRS-UCP and the project Labex MME-DII (ANR11LBX0023-01

\section{REFERENCES}

[1] Julien Abrossimoff, Alexandre Pitti, and Philippe Gaussier. Visual learning for reaching and body-schema with gain-field networks. 2018 Joint IEEE 8th International Conference on Development and Learning and Epigenetic Robotics (ICDL-EpiRob), 2018.

[2] Garrett E Alexander. Biology of parkinson's disease: pathogenesis and pathophysiology of a multisystem neurodegenerative disorder. Dialogues in clinical neuroscience, 6(3):259, 2004.

[3] John M Allman, Atiya Hakeem, Joseph M Erwin, Esther Nimchinsky, and Patrick Hof. The anterior cingulate cortex: the evolution of an interface between emotion and cognition. Annals of the New York Academy of Sciences, 935(1):107-117, 2001.

[4] Alan Baddeley. Working memory. Science, 255(5044):556-559, 1992.

[5] David Badre. Opening the gate to working memory. Proceedings of the National Academy of Sciences, 109(49):19878-19879, 2012.

[6] Apoorva Bhandari and David Badre. Learning and transfer of working memory gating policies. Cognition, 172:89-100, 2018.

[7] M. Botvinick, T.S. Braver, D.M. Barch, C.S. Carter, and J.D. Cohen. Conflict monitoring and cognitive control. Psychological Review, 108:624-652, 2001.

[8] George Bush, Phan Luu, and Michael I Posner. Cognitive and emotional influences in anterior cingulate cortex. Trends in cognitive sciences, 4(6):215-222, 2000.

[9] Christopher H Chatham and David Badre. Multiple gates on working memory. Current opinion in behavioral sciences, 1:23-31, 2015.

[10] Roshan Cools, Roger A Barker, Barbara J Sahakian, and Trevor W Robbins. Mechanisms of cognitive set flexibility in parkinson's disease. Brain, 124(12):2503-2512, 2001

[11] Mario Dipoppa, Marcin Szwed, and Boris S Gutkin. Controlling working memory operations by selective gating: the roles of oscillations and synchrony. Advances in cognitive psychology, 12(4):209, 2016.

[12] Alain Droniou, Serena Ivaldi, and Olivier Sigaud. Learning a repertoire of actions with deep neural networks. In 4th International Conference on Development and Learning and on Epigenetic Robotics, pages 229-234. IEEE, 2014.

[13] Kimberlee DArdenne, Neir Eshel, Joseph Luka, Agatha Lenartowicz, Leigh E Nystrom, and Jonathan D Cohen. Role of prefrontal cortex and the midbrain dopamine system in working memory updating. Proceedings of the National Academy of Sciences, 109(49):19900-19909, 2012.

[14] NF Forbes, LA Carrick, AM McIntosh, and SM Lawrie. Working memory in schizophrenia: a meta-analysis. Psychological medicine, 39(6):889-905, 2009 
[15] Adam Gazzaley, Jesse Rissman, and Mark Desposito. Functional connectivity during working memory maintenance. Cognitive, Affective, \& Behavioral Neuroscience, 4(4):580-599, 2004.

[16] Patricia S Goldman-Rakic. The physiological approach: functional architecture of working memory and disordered cognition in schizophrenia. Biological psychiatry, 46(5):650-661, 1999.

[17] David A Grant and Esta Berg. A behavioral analysis of degree of reinforcement and ease of shifting to new responses in a weigl-type card-sorting problem. Journal of experimental psychology, 38(4):404, 1948.

[18] JY Guo, John D Ragland, and Cameron S Carter. Memory and cognition in schizophrenia. Molecular psychiatry, 24(5):633-642, 2019.

[19] Michael E Hasselmo and Chantal E Stern. A network model of behavioural performance in a rule learning task. Philosophical Transactions of the Royal Society B: Biological Sciences, 373(1744):20170275, 2018.

[20] D. O. Hebb. The Organization of Behavior: A Neuropsychological Theory. Mahwah, NJ: Lawrence Erlbaum Associates, 1949.

[21] Michael J Higley and Marina R Picciotto. Neuromodulation by acetylcholine: examples from schizophrenia and depression. Current opinion in neurobiology, 29:88-95, 2014.

[22] R. Memisevic. Learning to represent spatial transformations with factored higher-order boltzmann machines. Neural Computation, 22:1473$1493,2010$.

[23] Luis Montesano, Manuel Lopes, Alexandre Bernardino, and José SantosVictor. Learning object affordances: from sensory-motor coordination to imitation. IEEE Transactions on Robotics, 24(1):15-26, 2008.

[24] Yuko Munakata, Seth A Herd, Christopher H Chatham, Brendan E Depue, Marie T Banich, and Randall C OReilly. A unified framework for inhibitory control. Trends in cognitive sciences, 15(10):453-459, 2011.

[25] Alex Pitti, Raphaël Braud, Sylvain Mahé, Mathias Quoy, and Philippe Gaussier. Neural model for learning-to-learn of novel task sets in the motor domain. Frontiers in psychology, 4:771, 2013.

[26] Charan Ranganath, Michael X Cohen, Cathrine Dam, and Mark D'Esposito. Inferior temporal, prefrontal, and hippocampal contributions to visual working memory maintenance and associative memory retrieval. Journal of Neuroscience, 24(16):3917-3925, 2004.

[27] Nicolas P Rougier, David C Noelle, Todd S Braver, Jonathan D Cohen, and Randall C O'Reilly. Prefrontal cortex and flexible cognitive control: Rules without symbols. Proceedings of the National Academy of Sciences, 102(20):7338-7343, 2005.

[28] Wolfram Schultz, Peter Dayan, and P Read Montague. A neural substrate of prediction and reward. Science, 275(5306):1593-1599, 1997.

[29] Jun Tanji and Eiji Hoshi. Behavioral planning in the prefrontal cortex. Current opinion in neurobiology, 11(2):164-170, 2001.

[30] Tomer Ullman, Andreas Stuhlmüller, Noah Goodman, and Joshua B Tenenbaum. Learning physics from dynamical scenes. In Proceedings of the 36th Annual Conference of the Cognitive Science society, pages $1640-1645,2014$

[31] Theodore P Zanto, Michael T Rubens, Arul Thangavel, and Adam Gazzaley. Causal role of the prefrontal cortex in top-down modulation of visual processing and working memory. Nature neuroscience, 14(5):656, 2011. 